\title{
LA RIQUEZA MUNDIAL ALCANZA PARA TODOS
}

\author{
Juan Manuel Díaz Yarto \\ CEPHCIS \\ UNIVERSIDAD NACIONAL AUTÓNOMA DE MÉXICO \\ Mientras los leones no tengan sus propios historiadores, \\ las historias de cacería siempre glorificarán al cazador \\ Proverbio africano
}

\section{RESUMEN}

Se hace una revisión de la manera en que el pensamiento económico hegemónico producido en los centros académicos de los países desarrollados comprende la participación e inclusión de los mercados latinoamericanos en la economía globalizada. El artículo revisa, desde el pensamiento crítico, los procedimientos económicos seguidos por el mercado global en el proceso de incorporación de las economías de América Latina y las consecuencias que ha acarreado la indiscriminada apertura de las economías nacionales al capital internacional. Además, se ponen en tensión las posibilidades reales de desarrollo mundial que el capitalismo moderno plantea a partir del maridaje de la tecnología y las comunicaciones, con el desigual e inequitativo funcionamiento de la economía global.

Palabras clave: modernidad, desarrollo, desigualdad. 


\begin{abstract}
The author reviews the way in which hegemonic economic thought produced in academic centers in developed countries considers the participation and inclusion of Latin American markets in the globalized economy. From a perspective of critical thought, the author reviews the economic procedures followed by the global market in the process of incorporating Latin American countries, and the consequences from the indiscriminate opening of their national economies to international capital. In addition, the article questions the possibilities for world development as proposed by modern capitalism on the basis of the union between technology and communication, given the unequal, unfair way in which the global economy functions.
\end{abstract}

Key words: modernity, development, inequality. 
La centralidad e «inevitabilidad» de la globalización, ${ }^{1}$ así como la comprensión de que esta sea un proyecto de clase, son dos ángulos de mirada de este tema crucial en la discusión sobre los alcances de la economía del mundo moderno. Aunque en el presente artículo no evaluaré el contenido intelectual de las mencionadas posiciones, sí deseo resaltar el que ambas coinciden en un punto trascendente, que el momento del desarrollo capitalista actual arrastra una contradicción fundamental en el sistema de producción de riqueza y explotación entre los países que están dentro del orden mundial y los que permanecen fuera. Contradicción que impulsa fuerzas que obstruyen el normal funcionamiento de los mecanismos de producción y de acumulación sistémica. Esta contradicción, que se extiende a todos los países, se aparece como la lógica interna de un sistema mundial capaz de controlarlo todo; con suficientes recursos económicos y financieros que aportan un mejor nivel de vida a la población más rica del mundo. ${ }^{2}$

Desde la revolución industrial inglesa es nítida la prevalencia de los intereses económicos de los países dominantes —en su forma moderna, no colonial— en la configuración del orden internacional de cada época. En esencia, este último constituye un entramado institucional que a la par de servir a los intereses de esas economías, atiende a la necesidad inescapable de ordenar el mundo en torno a reglas que han de respetarse a fin de hacer posible la convivencia entre naciones. Los paradigmas económicos y políticos resultantes concuerdan con la visión y objetivos de la nación líder o de las naciones líderes y, con frecuencia, obligan a los estados periféricos a absorber disonancias históricas e implantar cambios que no corresponden a su evolución social o intereses (Ibarra 2005:7-8).

Sin pretender minimizar las consecuencias sociales producidas por esta desigual repartición de la riqueza, mi interés se centra ahora en reflejar la indiscutible capacidad productiva del trabajo y la incapacidad de los grupos privilegiados para tomar 
conciencia de lo crítico de esta realidad. Esto se debe a que la velocidad de movimiento de los flujos financieros deja al ritmo del proceso de producción material sin posibilidad de competir en el nivel de capitalización de la inversión, aunque estén aún fuertemente relacionados en ciertas circunstancias, como puede ser una caída en los mercados financieros o en la bolsa de valores. ${ }^{3}$ La modernidad del sistema por su velocidad y volumen ha ofrecido a los dueños del capital posibilidades de tener acceso inmediato a más lugares para operar trasferencias, pero también ha profundizado la división internacional del trabajo repartiendo la producción en diversas naciones del tercer mundo (Ibarra 2005).

El primer mundo modernizado se ha quedado solo en la centralidad y regulación del sistema capitalista y ha conseguido robustecerse cuantitativamente en comparación con épocas anteriores, a cambio de hincar la desigualdad e inequidad en los países modernamente pobres del tercero. Esto no quiere decir que este proceso responda únicamente a un proyecto ideológico estructurado que no toma en cuenta el proceso de desarrollo natural, ni tampoco que la globalización solamente aparezca planificadamente en función de los intereses sistémicos. De tal manera que si pudiera resumir el planteamiento central de este ensayo, debería decir: primero, que la modernidad del modelo productivo y financiero ${ }^{4}$ expansionista tiene su triebkraft, fuerza motriz, en el éxito productivo del desarrollo científico y tecnológico; segundo, que la modernidad vista como desarrollo económico en las naciones del tercer mundo responde a procesos productivos combinados: endógenos y exógenos; y tercero, que la riqueza producida mundialmente, como resultado de la globalización de la modernidad del sistema capitalista, es posible que sea capaz de satisfacer las necesidades primarias y de crecimiento de todos los países. 
La acepción de globalización que enmarca este artículo pretende ser lo más general e inclusiva posible, para lo que he adoptado la propuesta de Octavio Ianni:

\begin{abstract}
Aunque la nación y el individuo sigan siendo muy reales, incuestionables y estén presentes todo el tiempo, en todo lugar, y pueblen la reflexión y la imaginación, ya no son «hegemónicos». Han sido subsumidos formal o realmente por la sociedad global, por las configuraciones y los movimientos de la globalización. El mundo se ha mundializado, de tal manera que el globo ha dejado de ser una figura astronómica para adquirir más plenamente su significación histórica (Ianni 1999: 3).
\end{abstract}

\title{
LA MODERNIDAD CAPITALISTA
}

La ciencia económica moderna ${ }^{5}$ escrita en los países dominantes del primer mundo ha construido un lenguaje técnico que explica a los ciudadanos de todas las naciones las condiciones que son necesarias para formar parte de la universalización del sistema económico capitalista moderno. «Desde que se formó el moderno capitalismo, el mundo pasó a ser influido por el patrón de racionalización generado como una cultura de ese mismo capitalismo» (Ianni 2005: 92). Esta argumentación se sostiene en las categorías de dinero - libre flujo del capital financiero—, mercancía — libre intercambio de bienes y servicios - y tecnología — libertad de información mundial— como ejes estratégicos del sistema. Este mensaje de bienestar global ha sido traducido a todos los idiomas y se ha enraizado en el pensamiento económico de los países menos desarrollados que aceptan simultáneamente la hegemonía metodológica que marca el camino para alcanzar los retos y las metas económicas modernas.

El hecho de que el capitalismo se vuelva el modo de producción dominante en los países hoy atrasados, precisamente cuando el sistema se desplaza de la fase propiamente competitiva a la 
monopolista, no es una cuestión secundaria o incidental: es una confluencia histórica decisiva, que en buena medida determinará la suerte del capitalismo latinoamericano (Aguilar 2005: 198).

Este lenguaje nos ha preparado para habitar un mundo en el que nos resultan conocidos y hasta familiares procesos como: la fusión de la tecnología, la técnica y la información, la internacionalización del mercado y de las mercancías, la libre movilidad del capital financiero por encontrar las más óptimas plazas para reproducirse, y la recomposición de los monopolios y de las trasnacionales. La mayoría de los habitantes del planeta somos, a través de los medios de comunicación, espectadores no participantes de los procesos globales de la economía e inconcientemente hemos aprendido a identificar la idea de sociedad avanzada con la de capitalismo moderno, y a estas dos con la acepción general de modernidad. Tanto es así que nuestra percepción del mundo, lejos de angustiarse frente a su complejidad conceptual, es la de un tema de fácil comprensión al alcance de cualquier inteligencia.

Otra particularidad de esa modernidad actual es que está siendo objeto de estudio por parte de especialistas de diferentes áreas del conocimiento como la sicología, sociología, historia, economía, etc., al grado de darnos cuenta que estamos frente a un proceso que tiene que ver con todos los espacios de nuestra vida social y privada. Es decir, el concepto se ha popularizado al grado de que se puede ser moderno hasta para lo más tradicional, por ejemplo: la novel cocina mexicana; la incorporación del adobe antiguo en la arquitectura moderna; la modernidad en el diseño del vestido tradicional, etc. Es importante recordar que el conocido economista Xavier Sala-iMartín $^{6}$ afirma que la globalización ha acentuado los regionalismos en el sentido de que, en la mayoría de las capitales del mundo, podemos encontrar restaurantes con comida griega, italiana, china, mexicana, argentina, etcétera. 
Existen otras voces, como la del filósofo Bolívar Echeverría, quien afirma que la modernidad es un proceso que apenas está por alcanzarnos, diciendo que:

\footnotetext{
... está aún por hacerse [y] este concepto se refiere a un sistema civilizatorio desarrollado en la sociedad occidental, el cual intenta incluir en el funcionamiento de la vida cotidiana los impactos de una revolución técnica y tecnológica de gran alcance, que comenzó de forma temprana - tal vez en el siglo XI- con muchos episodios $\mathrm{y}$, sobre todo, acelerado con la Revolución Industrial en el XIX (Echeverría 2005: 4).
}

A pesar de la innumerable existencia de opiniones sobre este tema, me parece importante reflexionar alrededor de la siguiente pregunta: ¿De qué hablamos cuando pensamos en la modernidad económica?

El pensamiento económico formal ha funcionado adecuadamente para inventar mecanismos de medición del grado de desarrollo de los pueblos o para evaluar el nivel y calidad de vida de las personas en las sociedades desarrolladas. Desde la cúpula del pensamiento central-capitalista, después de la Segunda Guerra Mundial, los países fueron ordenados en tres mundos económicos, tomando como punto de partida su capacidad económica para cumplir con tasas de producción y de ganancia establecidas por los países occidentales desarrollados, a saber: primer bloque, los países desarrollados de occidente más Japón; segundo bloque, los países socialistas, y tercer bloque, o bien tercer mundo, todos los demás. Este ordenamiento decidió el lugar que ocuparon los países en la distribución internacional del trabajo y al mismo tiempo condicionó sus posibilidades de desarrollo e integración a las necesidades del mercado en correspondencia con el bloque político al que pertenecían. La reglamentación eurocéntrica funcionó durante casi treinta años subordinada a principios ideológicos y políticos antes que económicos, hasta que la incorruptible realidad volvió a hacer de las 
suyas exigiendo nuevas herramientas teóricas para actualizar la reclasificación de los países.

Con la desaparición del bloque socialista ya no hubo segundo mundo, pero esta nueva situación mundial no evitó que algunos países continúen enmarcados en la categoría de tercer mundo; se constituye tan diversa e indefinida que agrupa, irresponsablemente, realidades de países económicamente distintos y distantes que ni siquiera comparten parámetros culturales. Al mismo tiempo, como rasgo sobresaliente de esta modernidad, se ha hecho evidente que dentro de los países del primer mundo existen realidades propias del tercero, ${ }^{7}$ así como en el tercero las hay del primero, lo que ha venido a derrumbar la idea de un sistema capitalista económico integral y ha demostrado, por vez primera, que los «zapatos viejos» ${ }^{8}$ de la economía no pertenecían solamente al mundo pobre, sino que han sido una condición de vida de la economía impuesta por la modernidad.

De hecho, los pobres en Estados Unidos suman 37 millones, y representan un $12.6 \%$ —apenas una décima por debajo de 2004 — del total de la población del país, según datos de la Oficina del Censo de Estados Unidos. La cifra sorprende si se piensa que EE.UU. es una potencia económica mundial, con un ingreso familiar medio de US\$46.300 anuales y séptimo en el ranking del índice de desarrollo humano del Programa de Naciones Unidas para el Desarrollo, PNUD, lo que lo ubica entre los mejores lugares del planeta para vivir y prosperar. Cerca de un cuarto de la población negra, un 21,8\% de los hispanos y el 8,3\% de los blancos americanos viven en la pobreza. Los datos más recientes de la Oficina del Censo arrojan que la pobreza en Estados Unidos tiene color y edad. Entre los más pobres están los inmigrantes, la población afro-americana y los niños. La tasa de pobreza infantil alcanza el 17,6\%, o 12,9 millones de niños, de los cuales el $28,3 \%$ pertenece a los hispanos, el 34,5\% a los afro-americanos y un $10 \%$ a los blancos estadounidenses (Martínez 2006). 
Los países desarrollados observaron que es muy comprometido, costoso, lento y sin garantía de éxito, impulsar directamente, con presencia física de capital o de fuerza, cambios estratégicos, considerados económicamente óptimos, en los países atrasados, por lo que decidieron modificar los principios universales con los que comprometían a las naciones subdesarrolladas en el orden mundial. A partir de los años setenta, los países se repartieron nuevamente en dos grupos simples: los desarrollados y los que están en vías de desarrollo. Con esta reclasificación se ha impuesto otra manera de comprender el mundo a partir de un reordenamiento de categorías sociales, políticas y económicas. Las sociales fueron ordenadas bajo la pobreza y la desigualdad, las políticas se resumieron en países democráticos y no democráticos y las económicas en producción y distribución de la riqueza.

\footnotetext{
La actual etapa de desarrollo capitalista se enmarca en el proceso de expansión del gran capital internacional financiero, así como por la dislocación de los procesos productivos desde los países centrales a la periferia, posible de realizar gracias a las favorables condiciones de inversión ... y los menores costos de producción (Saxe-Fernández y N. R. Omar 2001: 93).
}

El parámetro con el que se comenzó a medir la diferencia en el nivel de desarrollo de los países fue la capacidad de inversión e innovación en tecnología y técnica, I+D. ${ }^{9}$

Del total del monto de la inversión mundial en investigación y desarrollo, el $42 \%$ va a los Estados Unidos y a Canadá, el 28\% a Europa, el 27\% a los países asiáticos, y solo el $1 \%$ a América latina, según la Red Iberoamericana de Indicadores de Ciencia y Tecnología, Ricyt. Mientras Israel gasta el 5\% de su producto bruto en investigación y desarrollo, Japón gasta más del 3\%; los Estados Unidos cerca del 3\%; Corea del Sur, el 2,5\%; China, el 1,2\%, y la India cerca del 1\%. El promedio de América Latina es del 0,5\%, según el Programa de Desarrollo de las Naciones Unidas, PNUD. Latinoamérica se está quedando atrás porque no logra atraer 
inversiones privadas en investigación, según muestran los estudios cerca del $70 \%$ de las investigaciones hechas en México y Argentina están financiadas por el gobierno, mientras que el porcentaje es de menos del 35\% en Estados Unidos; el 25\% en Corea del Sur, y el 18\% en Japón (Oppenheimer 2005).

En la época de la globalización — esto es a partir de los años ochenta- el mundo se dividió, desde la teoría, nuevamente en dos mitades:

a) los países que viven la modernidad desarrollando e incorporando tecnología a sus procesos productivos; y

b) aquellos que están siempre en camino de alcanzarla y que terminan funcionando como consumidores de tecnología de segunda o desecho.

Con esta estrategia teórico-práctica de conceptualización del mundo, el concepto de modernidad fue puesto nuevamente al alcance de cualquier país —productor o comprador de tecnología- que tuviera la necesidad económica de adoptar el reglamento general exigido centralmente para alcanzarla, sin importar la intención política, o sea:

En asuntos de política económica, las buenas intenciones son cosa subjetiva, no interesan; lo que cuenta son los resultados concretos de la política trazada, su aspecto objetivo ... El capital extranjero mantiene un especial estado de conciencia que predispone a la entrega o a la sumisión. Este estado de conciencia invade todos los rincones del país, todos los sectores sociales actuantes económica y políticamente; se refleja en todos los aspectos de la vida nacional, como si fuese un fatalismo histórico frente al cual no hubiese otra alternativa que inclinarse (Gunder 2005). 
En los hechos, este complejo proceso de readaptación a la economía mundial fue estableciendo condiciones estrictas para que cada país adoptara una versión del paquete de medidas dictadas por los asesores del Banco Mundial y del Fondo Monetario Internacional, y con ello situarse en la división internacional del trabajo.

... las fuerzas ... de la sociedad global actúan, sobredeterminado heteronómicamente [sic], erosionando las capacidades de modificar la posición que se tiene en el sistema interestatal o ante los grandes corporativos multinacionales. El lugar que se ocupa en la escala jerárquica de la aldea global reserva a nuestros países [latinoamericanos] la condición de provincias de la misma (Gandarilla 2004: 54).

Lo dicho hasta aquí es el marco para introducir la idea central del presente artículo: la economía moderna tiene un lindo y bien diseñado traje moderno, pero se sostiene sobre unos zapatos viejos que no corresponden con su apariencia. Con el traje presume los alcances del mercado, los beneficios del ritmo financiero y de los avances tecnológicos. Sin embargo, no puede evitar calzar zapatos viejos para moverse en algunas realidades con más dificultades que en otras $\mathrm{y}$, con esta actitud, da cuenta del crítico, desigual e injusto mundo en que vivimos.

El capitalismo moderno se viste con el luminoso traje de la globalidad que le ha funcionado para instrumentar tanto las variables económicas que impulsan el bienestar, como aquellas que lo frenan. Esta contradicción es cada vez más compleja pues el sistema no modifica su voraz necesidad de expropiación y apropiación de riqueza.

Me referiré a dos características que describen sus zapatos viejos. La primera, se refiere a la manera en que problemas económicos y sociales como la desigualdad, la inequidad y la injusticia, han tenido y tienen una indiscutible presencia histórica en la esencia del proceso productivo del capitalismo. La otra, consecuencia de la anterior, es 
el desinterés de los centros económicos y financieros mundiales por resolver definitivamente estas deficiencias sistémicas convirtiéndolas en un nudo problemático que:

a) limita la flexibilidad y el dinamismo de las economías en países pobres para inventar alternativas efectivas de desarrollo;

b) produce un patrón de comportamiento deficiente, en los actores responsables, que garantiza la imposibilidad de cambios esenciales locales en el corto plazo.

\section{LA REALIDAD ECONÓMICA DEL MUNDO}

Como dije antes, la categoría modernidad es usada por el pensamiento económico hegemónico para descalificar lo no moderno, lo viejo o lo pasado. La intención directa es la de cerrar la posibilidad de ser moderno a todo lo que no pertenezca al mundo desarrollado; es una categoría que establece un cerco geográfico y comercial alrededor de algunos países en los que funciona la legalidad, los derechos humanos, la racionalidad, la educación, la salud y el estado de derecho. En esta región la modernidad refleja grandes logros en áreas tan importantes como:

- la tecnológica, que permite tener mejores hospitales, medios de transporte, instrumentos de trabajo y de educación, así como aparatos que hacen más cómoda la vida personal — teléfonos celulares, agendas electrónicas, etc.;

- la biotecnológica y la nanotecnológica, que sin duda son las que definirán la economía del siglo que viene en cuanto a alimentación, agricultura, medicina, electrónica, informática, materiales y manufacturas; y a la que le están apostando grandes empresas trasnacionales como Exxon, Mobil, IBM, Dow 
Chemicals, Seros, 3M, Alcan Aluminium, Johnson y Johnson, Hewlet Packard, Motorola, Sony, Toyota y Hitachi;

- la genética y la biogenética, para el potencial desarrollo del ser humano como especie;

- el impulso a la ciencia, que se traduce en investigación y desarrollo, I+D, y que permite perfeccionar la maquinaria industrial y agrícola, optimizar el rendimiento en el uso de los recursos, elevar la rentabilidad del capital invertido, mejorar la planeación estratégica y prospectiva de modelos económicos;

- el despegue de las comunicaciones satelitales y el uso de la fibra óptica como conductor de alta velocidad.

Resumiendo, la modernidad significa la inserción en la sociedad de procesos de innovación que aportan, cada vez más, mejores condiciones e instrumentos para alcanzar una vida moderna con bienestar económico. Estos representan, al mismo tiempo, exigencias a cumplir para aquellos países con problemas de crecimiento y desarrollo económicos, que además enfrentan la desigualdad en los mecanismos internacionales de intercambio y la inequidad financiera de las inversiones internacionales como prácticas globales de los mecanismos de mercado de los países centrales. La desigualdad y la inequidad son categorías propias de las economías subdesarrolladas y, desde luego, políticamente incorrectas desde la eurocéntrica óptica de las economías desarrolladas.

Los entornos y las experiencias modernos atraviesan todas las fronteras de la geografía y la etnia, de la clase y la nacionalidad, de la religión y la ideología: se puede decir que en este sentido la modernidad une a toda la humanidad. Pero es una unidad paradójica, la unidad de la desunión: nos 
arroja a todos en una vorágine de perpetua desintegración y renovación, de lucha y contradicción, de ambigüedad y angustia (Ianni 1999: 137).

Aquel país que mantenga una política económica incapaz de utilizar eficientemente los instrumentos de la globalización — libre flujo de mercancías, libre flujo de capitales e integración comercial- pertenece al mundo de lo inacabado, de lo desigual, de lo antidemocrático, de lo no moderno. La modernidad globalizada requiere para su funcionamiento del establecimiento de gobiernos democráticos en todo el mundo que cumplan cabalmente con los principios exigidos por las centralizadas democracias occidentales y que agilicen el beneficio y la reproducción de las variables económicas internacionales.

En el seno de esta conformación [alianza Estado-capital], en su doble carácter, como interiorización del capitalismo en el Estado y como reorganización política del mundo, la realidad de los Estados nacionales como nodos diseminados de una red de poder global —que nace y recrea permanentemente su condición primigenia de colonialidad—, permite una mayor capacidad de reproducción sistémica (Gandarilla 2005: 39-40).

Esta estrategia se descubre cuando se analiza el interés y rumbo de los flujos de capital de la Inversión Extranjera Directa, es decir que este capital llega a los países con la intención de aumentar su capacidad financiera, por lo que se dirige a aquellos países que estén en condiciones de garantizar esta condición. Tal realidad me permite decir que las globalmente redituables posiciones económicas, financieras y comerciales, son permanentemente ocupadas por los países centrales, quienes depositan sus capitales en aquellos otros tradicionalmente, pero también, temporalmente seguros (ver Tabla 2 y gráficas anexas). 
Aunque en los últimos años algunas de estas cifras indican una tendencia generalizada a la baja de la participación de Latinoamérica en la captación de recursos financieros, que podría ser indicio de un reencauzamiento del capital hacia otras regiones del mundo, los 68,000 millones de dólares invertidos en la región en 2005 aún significan $11 \%$ más que el año anterior, sin olvidar que se debieron, casi únicamente, a los depositados como inversiones en los centros financieros extraterritoriales de la región. Sin contar lo extraído en estos centros, las salidas de capital ${ }^{10}$ se elevaron a 43,000 millones de dólares, es decir un aumento de $125 \%$ en relación con $2005,{ }^{11}$ en donde Brasil encabeza la lista seguida por México, Chile, Venezuela y Argentina (se sugiere revisar el World Investment Report 2007).

Esta capitalización de las inversiones no quiere decir que el país que la recibe experimente periodos de desarrollo económico estables a mediano plazo, ni tampoco implica la renovación de la tecnología instalada al interior de sus procesos productivos, sino que más bien da cuenta del tamaño de la desequilibrada extracción y apropiación de riqueza por una parte del mundo — como se muestra en las gráficas anexasindependientemente de si estos movimientos financieros inciden o no en el desarrollo económico del país huésped.

La globalidad del mercado también ha confundido la identidad de los sujetos sobre sus saberes locales en los países menos desarrollados al confrontarlos de golpe con los sentidos comercial y cultural del consumo. Por un lado, el comercio internacional es una necesidad insustituible para el funcionamiento de las economías nacionales. La necesaria y mal estructurada inserción en la globalidad, común en los tratados de libre comercio en la región, no ha hecho más que arrasar con los proyectos nacionales de desarrollo del mercado interno. La premura con la que se decidió abrir unilateralmente las fronteras a las mercancías y al capital mundial provocó la fractura de 
procesos económicos locales. Por otro lado, el discurso de la globalización argumentó, con enorme efectividad, que los obstáculos que hacen imposible la modernización de la vida local provienen de los saberes culturales tradicionales de los pueblos; y con esta afirmación convenció a las sociedades de la urgencia de modernizar ese conocimiento con el fin de incorporarlo al consumo y al uso de la cultura del mundo moderno.

La tecnificación de las relaciones sociales, en todos los niveles, se universaliza. En la misma proporción en que se da el desarrollo extensivo e intensivo del capitalismo en el mundo, se generalizan la racionalidad formal y real inherente al modo de operación del mercado, de la empresa, del aparato estatal, del capital, de la administración de las cosas, gentes e ideas, todo codificado ... ahí se unen el derecho y la contabilidad, la lógica formal y la calculabilidad, la racionalidad y la productividad, de tal manera que en todos los grupos sociales e instituciones, en todas las acciones y relaciones sociales, tienden a predominar los fines y valores constituidos en el mercado... (Ianni 1999: 9).

Ajenos a toda responsabilidad histórica los gobernantes de los países desarrollados afirman que en los países en vías de desarrollo existen grupos de ciudadanos de segunda clase, personas que se niegan a comprender la importancia de hacer un esfuerzo por parecerse al modelo de ciudadano que habita en los países del primer mundo. Desde este saber globalizado se decide que estos grupos de personas son prescindibles para el mundo moderno. Representan el costo que tienen que pagar las nuevas democracias para transitar por el camino de la modernidad. Por ello no debe sorprendernos que el proteccionismo económico practicado por los mercados de los países desarrollados descalifique esta práctica para las economías débiles y les imponga condiciones desiguales de intercambio. Esta actitud debería sorprender más, sobre todo cuando es 
tan pronunciada la diferencia de PIB por habitante de América Latina, que apenas creció $1.2 \%$ de 1990 a 2006, por debajo del promedio mundial de $1.3 \%$.

Tabla 1. Crecimiento de PIB, 1971-2006, América Latina

\begin{tabular}{|l|c|c|c|c|c|c|}
\hline & $1971-80$ & $1981-89$ & $1990-97$ & $1998-2003$ & $2004-06$ & $1990-06$ \\
\hline Argentina & 2,8 & $-1,0$ & 5,0 & $-1,3$ & 8,7 & 3,3 \\
\hline Brasil & 8,6 & 2,3 & 2,0 & 1,5 & 3,4 & 2,1 \\
\hline Chile & 2,5 & 2,8 & 7,0 & 2,7 & 5,5 & 5,2 \\
\hline Colombia & 5,4 & 3,7 & 3,9 & 1,1 & 4,8 & 3,0 \\
\hline México & 6,5 & 1,4 & 3,1 & 2,9 & 4,0 & 3,2 \\
\hline Perú & 3,9 & $-0,7$ & 3,9 & 2,0 & 6,0 & 3,6 \\
\hline Uruguay & 2,7 & 0,4 & 3,9 & $-2,1$ & 8,6 & 2,5 \\
\hline Venezuela & 1,8 & $-0,3$ & 3,8 & $-2,7$ & 12,2 & 2,9 \\
\hline A.L. Total & 5,6 & 1,3 & 3,2 & 1,3 & 5,2 & 2,9 \\
\hline Per cápita & 3,0 & $-0,8$ & 1,4 & $-0,3$ & 3,6 & 1,2 \\
\hline Por trabajador & 1,7 & $-1,5$ & 0,5 & $-1,1$ & 2,8 & 0,3 \\
\hline Fuente: Ffrench Davis 2007 & & & & & \\
\hline
\end{tabular}

Fuente: Ffrench Davis 2007.

Estos indicadores demuestran el hecho de que América Latina no ha avanzado de manera importante en el fortalecimiento de su economía y que, si comparamos con los países del primer mundo, el PIB de la región es solo una cuarta parte del registrado por éstos - 35, 782 vs 8.392 millones de dólares-. Los porcentajes observados en la tabla provienen del establecimiento de un modo de producción mundial controlado centralmente, que ha producido, en el ámbito regional y local, la implementación de políticas económicas que no solucionan de manera integral los problemas particulares de equidad y desarrollo, esto es: elevar el bienestar del conjunto de la población y 
avanzar significativamente en la consolidación de economías dinámicas y competitivas (Ffrench 2007).

\section{LA PRODUCCIÓN MUNDIAL Y SU DESIGUAL DISTRIBUCIÓN}

La modernidad técnica y tecnológica ha desarrollado instrumentos de producción que, en muchos casos, superan el rendimiento productivo de los sistemas nacionales, elevando las tasas de productividad a niveles nunca antes experimentados. La riqueza concentrada en algunas manos rebasa fácilmente el PIB de más de un país en vías de desarrollo, como lo demuestra un trascendente estudio de la Universidad de las Naciones Unidas, WIDER.

Utilizando tipos de cambio, la riqueza global de los hogares suma \$125 trillones [de dólares] en el año 2000, equivalente a tres veces el valor total de la producción global, PIB ... [vista a nivel personal tenemos que] $2 \%$ de los adultos más ricos en el mundo posee más de la mitad de la riqueza global de los hogares ... [Asimismo] $1 \%$ de adultos más ricos posee el $40 \%$ de los activos globales...y que el $10 \%$ de los adultos [en el mundo] cuenta con el $85 \%$ del total mundial. En contraste, la mitad más pobre de la población adulta del mundo solo es dueña del $1 \%$ de la riqueza global. A pesar de que en Norteamérica solo habita el $6 \%$ de la población adulta mundial, posee el $34 \%$ de la riqueza. Para estar entre el $10 \%$ más rico de los adultos en el mundo se requiere $\$ 61,000$ [dólares] en activos y más de $\$ 500,000$ para pertenecer a la del $1 \%$ más rico —37 millones de personas (WIDER-ONU 2006: 2-3).

Con esta información es posible apreciar que los países del tercer mundo son grandes productores de riqueza, pero también son grandes exportadores del capital que se acumula en las arcas de algunos inversionistas concentrados en los países desarrollados. Estos niveles de utilidad podrían, sin duda alguna, liberar al mundo de la injusta 
pobreza que fustiga a más de la mitad de la población mundial; pero no lo harán, pues esta solución no pertenece a la esencia económica del sistema capitalista mundial.

En los últimos 50 años la producción mundial de alimentos ha aumentado de forma vertiginosa, incluso más que la tasa de la población mundial. Entre 1990 y 1997 la producción per cápita de alimentos creció casi un 25\%, sin embargo, en el mundo aún pasan hambre 830 millones de personas, aproximadamente una de cada siete, lo cual representa a una población mayor a la que vive en Europa. El problema del hambre, como fenómeno grave y generalizado, no se debe a la escasez de alimentos, sino a la pobreza de las poblaciones afectadas, quienes carecen de los medios para adquirirlos (ONU 2007).

La modernidad asumida por el sistema ha puesto a los países en desarrollo en desventaja frente a sí mismos. Algunos instrumentos teóricos de la economía moderna, enmarcados en lo que Edgardo Lander califica como «colonialidad del saber» ${ }^{12}$ son utilizados para diseñar mundos perfectos en los que únicamente caben aquellos países o personas que cumplen y soportan estrictas medidas económicas.

El principio de la zanahoria y el garrote ya no define la política internacional practicada por los países eurocéntricos, en esta nueva época del desarrollo económico el cebo estará permanentemente delante de los países subdesarrollados, sin que puedan alcanzarlo, y estos pagarán por la oportunidad de continuar persiguiéndolo. Se puede decir, incluso, que estos países están convencidos de que los modelos de desarrollo propuestos desde el centro fueron diseñados para la solución de sus necesidades y que, en la medida que adoptaran las medidas y trasformaciones allí sugeridas, resolverían paulatinamente sus problemas de falta de desarrollo económico. La historia se encargó de desbaratar esa esperanza. Hoy, algunos países han aprendido que aquella infuncional propuesta de desarrollo que estuvo frente a sus ojos comparte, con la ahora renovada 
estrategia global de la economía moderna, el mismo principio económico, es decir: la extracción, concentración y centralización de la riqueza en unos cuantos países.

La modernidad perfecciona la forma de reproducción del capital pero no cambia su esencia. La modernidad es un concepto de plástico, limpio, brillante, rubio de ojos azules, atlético, fresco, experto en tecnología, competitivo y financieramente exitoso. Ningún país desea ser calificado de atrasado. La modernidad significa estar a la moda: moda es igual a ser moderno. Los jóvenes desean estar a la moda pues esta unifica e iguala visualmente; es un concepto que promete borrar las tradiciones culturales locales y las desigualdades regionales. La modernidad no hace referencia al pasado, se concibe a partir del futuro; ningún pasado es trascendente porque nada es tan importante como el hoy; el mensaje de moda nos dice que todos los seres son iguales y que tienen el mismo derecho a conseguir rápidamente su bienestar. Las drogas son el medio por el que la modernidad nos asegura la diversión; Internet es la modernidad en la educación, y los libros son vistos como el atraso y la lentitud. La espera no es un valor de los hombres modernos, la velocidad califica sus deseos de triunfar.

En la lógica primaria de los modernos instrumentos de dominación —medios de comunicación - la historia no es un argumento significativo y el futuro se puede ordenar a la carta. Los economistas nos encargamos de diseñar los requisitos económicos y financieros, así como las exigencias legales, correspondientes con el nivel de desarrollo económico de cada país. La modernidad nos enseña que el nivel de desarrollo depende de la disposición de cada país para adoptar el modelo de capitalismo en turno. 


\section{A MANERA DE CIERRE}

El capitalismo moderno no tiene interés por solucionar los problemas de inequidad social ni de desigualdad en la distribución de la riqueza amasada durante el histórico proceso económico de reproducción del sistema, debido a que no es capaz de mirar críticamente sus mecanismos de regulación, como tampoco es capaz de aceptar que es resultado de una historia plagada de conflictos sociales y económicos pendientes de analizarse cuidadosamente. El sistema capitalista moderno no tiene interés por aprehender las consecuencias negativas que ha impuesto la adopción normativa del modelo de producción dependiente sobre los países de Latinoamérica. El desconocimiento de las realidades específicas de los países del tercer mundo, así como las inadecuaciones en la aplicación de modelos económicos de desarrollo generales en América Latina, se definen como graves problemas de cálculo, y las crisis económicas producidas son justificadas por los organismos financieros internacionales, con el argumento de falta de capacidad local en la aplicación de las medidas recomendadas -por ejemplo: el consenso de Washington (1990) ${ }^{13}$ - a saber: disciplina presupuestaria, cambios en las prioridades del gasto público, reforma fiscal encaminada a buscar bases imponibles amplias y tipos marginales moderados, liberalización financiera, especialmente de los tipos de interés, búsqueda y mantenimiento de tipos de cambio competitivos, liberalización comercial, apertura a la entrada de inversiones extranjeras directas, privatizaciones, desregulaciones, garantía de los derechos de propiedad. En este sentido es una responsabilidad compartida, pues:

... el capitalismo no es un fenómeno de importación; no se produce simple e inopinadamente con la afluencia de capital extranjero, como expresión del desarrollo del imperialismo; es un fenómeno anterior y mucho más complejo en el que se funden, aunque sin llegar a sintetizarse en un todo integrado, por una parte la influencia del capitalismo a escala mundial ... y por otra la influencia 
del desarrollo capitalista interno ... que va volviéndose en modo de producción dominante... (Aguilar 2005: 201).

El perfil añejo del capitalismo moderno — de ahí sus zapatos viejos— está cada vez más determinado por la repetición de fallas en el análisis de los problemas regionales y locales, esto ha conducido a la decisión de poner en práctica modelos de desarrollo iguales para realidades diferentes. La exigencia esencial por la obtención de más poder y dinero ha funcionado históricamente como premisa económica para el desarrollo y crecimiento de los países centrales agrupados en bloques de intereses, y ha conseguido, en el largo plazo, empobrecer a más de la mitad de la población mundial, ${ }^{14}$ colocando de igual manera en una situación de riesgo la estabilidad del ecosistema del planeta.

Desde la óptica del saber globalizado construido y exportado por los pocos países que se benefician de la modernidad sistémica, la corrupción de algunos procesos económicos, sobre los que se sustenta su penetración en terceros países, no se aprecia como resultado de la concentración del poder por parte de los monopolios del mercado, ni de la presión financiera ejercida por intereses políticos de algunos grupos empresariales, sino que es descalificada como un rasgo característico del subdesarrollo contra el que no les corresponde actuar. En esta misma lógica las transacciones mundiales de capital y los paraísos fiscales no son un error del sistema, sino más bien un acierto bancario para que no se estanque la dinámica monetaria de la economía capitalista. Al decir de Alfredo Jalife-Rahme:

... en un solo año el DCC [Déficit de Cuenta Corriente] haya alcanzado el récord de 6.3 por ciento del PIB de Estados Unidos: un asombroso deterioro de 1.8 por ciento en un año que representa también el récord del lastre financiero para el resto del mundo. Estados Unidos requiere ahora 2 mil 900 millones de dólares de flujos externos de capital cada día para que la magia persista ... las 
grandes potencias se derrumban en forma típica cuando su alcance militar supera su poderío económico. Es justamente lo que vive Estados Unidos desde el 11 de septiembre de 2001: las guerras de Afganistán e Irak fueron los disparos de salva del inicio (Jalife-Rahme 2005).

Por otro lado, es un pensamiento inocente admitir que la naturaleza nos juega una mala pasada cuando se producen fenómenos climáticos con efectos devastadores sobre ciudades costeras o destruyen poblaciones enteras y obligan a los gobiernos a impulsar acciones paliativas, urgentes y costosas, que retardan la solución definitiva del grave problema económico-ecológico. Estos eventos ambientales nos sitúan frente a nuestra imposibilidad para:

- Analizar y aprehender, desde el ángulo de mirada de un pensamiento crítico, la historicidad económica de la especie humana.

- Construir una relación productiva positiva con la naturaleza y sustentabilidad de las materias primas.

- Desarrollar la capacidad para comprender e intervenir en el conocimiento y tratamiento de las causas que ocasionan la desigualdad e inequidad del sistema económico mundial.

La rápida revisión de algunos indicadores que dan cuenta de la gravedad alarmante del desastre ecológico que se está incrementando sirve para evidenciar la potencialidad de la crisis expuesta a lo largo del texto, a saber:

Cada día desaparecen para siempre 10 especies de seres vivos. Desde la época de la desaparición de los dinosaurios, 65 millones de años atrás, nunca se ha visto un exterminio tan rápido. Con esos seres vivos desaparece para siempre una biblioteca de conocimientos que la naturaleza sabiamente había acumulado; A partir de 1972 se han perdido cerca de 480 millones de toneladas de suelo 
fértil, una superficie equivalente a las tierras cultivables de India y Francia juntas, es decir que el $65 \%$ de las tierras que un día fueron cultivables, hoy ya no lo son; la mitad de las selvas existentes en el mundo en 1950 han sido deforestadas, por ejemplo, sólo en los últimos 30 años han sido talados 600 mil $\mathrm{km}^{2}$ de selva amazónica brasileña, el equivalente a la Alemania unida, o a dos veces la superficie de Zaire. Las inmensas reservas naturales de agua, formadas a lo largo de millones y millones de años, en este siglo pasado han sido sistemáticamente bombeados y están próximos a agotarse. El agua potable ya es uno de los recursos naturales más escasos, pues solamente el 0,7\% de toda el agua dulce es accesible al uso humano (Boff 2005).

Como se argumenta arriba, la concentración del capital internacional es una estrategia de enriquecimiento desarrollada y controlada por los países eurocéntricos ricos que arrebata a las naciones débiles su posibilidad de participar en el manejo de sus recursos. De ello nos ilustra el hecho de que

... durante los últimos años de la década de los noventa, se observaron un gran número de fusiones entre los grandes bancos comerciales, principalmente en Estados Unidos. Ejemplo de ello es la fusión de la compañía bancaria y de la firma financiera más grande de Estados Unidos, Citicorp con Travelers Group por más de 72,500 millones de dólares; la de BankAmerica y NationsBank, valoradas en 60,000 millones de dólares y la de Banc One Corp. First Chicago NBD, por cerca de 30,000 millones de dólares. Estas fusiones revelan una nueva etapa de consolidación bancaria en Estados Unidos. En Alemania también han ocurrido fusiones bancarias como la de Deutsche Bank y Dresdner Bank, operación que se calcula en 1,245 billones de dólares; en Japón destaca la fusión de los bancos Daiwa, Kinsi Osaka y Nara, que acumulan activos de aproximadamente 182.136 millones de dólares, así como la de los bancos Dai-Ichi Kangyo, Fujoi y Banco Industrial de Japón que en conjunto acumulan activos por 1,26 billones de dólares (Girón 2005). 
Los instrumentos económicos formales de la modernidad educativa estudian al hombre no para ofrecerle opciones eficaces que le ayuden a obtener mejor información sobre el mercado de bienes para alcanzar una mejor calidad de vida, sino para conocer la manera de comportarse en el mercado y así satisfacer sus necesidades y con ello condicionar y construir un saber global que lo convierta rápidamente en un consumidor dócil, compulsivo, ignorante y voraz. La antigua preocupación de la ciencia económica por edificar un estado de bienestar o por administrar eficientemente los bienes escasos del mundo para satisfacer las necesidades crecientes de la población y elevar su nivel de vida es letra muerta y no es reconocida por el pensamiento económico moderno. En la mayoría de las universidades se ha fusionado el estudio de la economía con el de administración de empresas, y ambas se han alejado del sentido humano. Así, también, el estudio de la ciencia económica se reduce al estudio y perfeccionamiento de modelos matemáticos que eleven los rendimientos financieros y productivos empresariales y que propongan mecanismos para reducir la participación del Estado en la atención de la pobreza, el desempleo y la seguridad.

El debate que me interesa plantear con este artículo, no es el que confronta el proteccionismo con el internacionalismo, o el localismo con la mundialización, sino el que se encamina a analizar la esencia normativa que regula y extiende los principios productivos de funcionamiento de la modernidad económica. La importancia de esta discusión no pretende escarbar en el qué o para quién se produce, sino en las raíces de cómo se implanta la modernidad del capitalismo; es decir, en indagar sobre las condiciones y relaciones de los factores productivos que conlleva la importación del modelo mundial de desarrollo.

Un mecanismo comúnmente utilizado para exportar las relaciones productivas es la mancuerna de la privatización con el comercio mundial, donde el derecho a la 
propiedad privada se considera un valor fundamental para el funcionamiento central del sistema $^{15} \mathrm{y}$, en consecuencia, se condena como una práctica desleal la toma de medidas proteccionistas comerciales por parte de las economías nacionales, de tal manera que no es ilógico el que las empresas trasnacionales instaladas en los países subdesarrollados mantengan deprimidas las condiciones laborales de sus empleados, esto es: el nivel de salarios y de prestaciones, el derecho de huelga, el impulso a la organización sindical, el derecho a vacaciones, etc. Estos actores económicos trasnacionales no se comportan con la misma trasparencia fiscal como lo hacen en sus países de origen, frente al pago de impuestos de terceros Estados, ni tampoco respetan las políticas de no agresión al medio ambiente, como lo exigen las leyes en los países centrales.

... estamos entrando en una nueva época de interdependencia, en la que las corporaciones sin Estado trascienden las fronteras nacionales, impulsadas por la tercera revolución tecnológica, y aligeradas por los nuevos sistemas de información. El contraste entre las premisas y las promesas de los teóricos de la globalización y las realidades contemporáneas no podría ser más marcado. En vez de naciones interdependientes, tenemos dramáticos contrastes entre naciones acreedoras y deudoras, corporaciones multimillonarias que se apropian de las empresas, intereses, regalías y excedentes comerciales, mientras que miles de millones de trabajadores y campesinos sobreviven en condiciones de pobreza y miseria (Petras y Veltmeyer 2003: 83).

El sistema capitalista mundial se encuentra entrampado en sus zapatos viejos, que cada vez más le impiden tener la sensibilidad y destreza necesarias para avanzar en el proceso de extracción de riqueza sin producir efectos económicos negativos en los países del tercer mundo que pongan en riesgo la frágil estabilidad del sistema en conjunto. 
Los economistas —en alianza con otros científicos— han demostrado poseer el conocimiento necesario para producir, con métodos sustentables, importantes cantidades de mercancías y trasportarlas de manera rentable allende los mares; para desarrollar la técnica y la tecnología que se traduzcan en deseables niveles de bienestar para cada vez más ciudadanos; y para producir insignes cantidades de riqueza que solucionen la inequidad, la desigualdad y la pobreza del mundo. Sin embargo, la aplicación de este conocimiento a los sistemas productivos no está en la esencia del capitalismo. De tal suerte que el sistema capitalista moderno continuará utilizando los mecanismos de regulación adecuados a las necesidades de acumulación eurocéntricas para solucionar problemas coyunturales — crisis sectoriales, crisis energéticas, crisis inmobiliarias, crisis de invasiones militares, etc. - , sin atender la creciente potencialidad de crisis financieras profundas, o las consecuencias económicas de la creciente confrontación de intereses comerciales regionales y locales. «Con la globalización, el mundo se polariza aceleradamente en otro sentido; hay regiones que prosperan, mientras otras decaen o se rezagan, hundiendo a sus poblaciones en descomposición social y conflictos distributivos cada vez más intensos» (Ibarra 2005: 23).

Por lo tanto la felicidad de unos pocos es insostenible en el mediano plazo si no solucionamos de manera definitiva la crisis mundial de crecimiento y desarrollo. 


\section{ANEXO DE TABLA Y GRÁFICAS}

Tabla 2. Afluencia de inversión extranjera directa por regiones y países principales (millones de dólares)

\begin{tabular}{|c|c|c|c|c|c|c|c|c|}
\hline Región/economía & $1970-1979$ & $\%$ & 1980-1989 & $\%$ & 1990-1999 & $\%$ & 2000-2004 & $\%$ \\
\hline Mundo & 241244,2 & & 938874,0 & & 4010278,7 & & 4219336,0 & \\
\hline Países desarrollados & 180086,3 & 74,6 & 724613,1 & 77,2 & 2669291,8 & 66,6 & 3100554,0 & 73,5 \\
\hline Alemania & 14363,9 & 6,0 & 15139,9 & 1,6 & 124615,0 & 3,1 & 263915,0 & 6,3 \\
\hline Australia & 10382,9 & 4,3 & 37180,0 & 4,0 & 62154,1 & 1,5 & 83776,0 & 2,0 \\
\hline Bélgica y Luxemburgo & 8651,2 & 3,6 & 22451,5 & 2,4 & 227093,0 & 5,7 & 176942,0 & 4,2 \\
\hline Canadá & 32346,2 & 13,4 & 37822,9 & 4,0 & 106090,9 & 2,6 & 128603,0 & 3,0 \\
\hline Dinamarca & 1078,6 & 0,4 & 2291,1 & 0,2 & 42604,9 & 1,1 & 43845,0 & 1,0 \\
\hline España & 5782,9 & 2,4 & 33814,4 & 3,6 & 105723,5 & 2,6 & 158951,0 & 3,8 \\
\hline Estados Unidos & 32190,0 & 13,3 & 336805,0 & 35,9 & 890636,0 & 22,2 & 697492,0 & 16,5 \\
\hline Francia & 14352,2 & 5,9 & 8180,6 & 4,1 & 226983,0 & 5,7 & 209577,0 & 5,0 \\
\hline Irlanda & 1370,2 & 0,6 & 2209,5 & 0,2 & 38946,1 & 1,0 & 100419,0 & 2,4 \\
\hline Italia & 5882,2 & 2,4 & 18872,7 & 2,0 & 39497,1 & 1,0 & 76020,0 & 1,8 \\
\hline Japón & 1240,0 & 0,5 & 1806,0 & 0,2 & 26318,8 & 0,7 & 37943,0 & 0,9 \\
\hline Países Bajos & 9578,3 & 4,0 & 27319,3 & 2,9 & 154328,3 & 3,8 & 155545,0 & 3,7 \\
\hline Reino Unido & 32571,8 & 13,5 & 103919,5 & 11,1 & 324768,9 & 8,1 & 294114,0 & 7,0 \\
\hline Suecia & 766,9 & 0,3 & 7063,8 & 0,8 & 130093,7 & 3,2 & 47808,0 & 1,1 \\
\hline Suiza & 0,0 & 0,0 & 9411,1 & 1,0 & 44418,6 & 1,1 & 55430,0 & 1,3 \\
\hline Paises en desarrollo & 61091,8 & 25,3 & 213561,7 & 22,7 & 1217689,9 & 30,4 & 1026116,0 & 24,3 \\
\hline América Latina y el Caribe & 32694,5 & 13,6 & 74381,1 & 7,9 & 444322,1 & 11,1 & 351580,0 & 8,3 \\
\hline Argentina & 1305,6 & 0,5 & 5844,0 & 0,6 & 68130,5 & 1,7 & 20874,0 & 0,5 \\
\hline Brasil & 12698,4 & 5,3 & 17214,2 & 1,8 & 99216,5 & 2,5 & 100136,0 & 2,4 \\
\hline Chile & 590,6 & 0,2 & 4376,1 & 0,5 & 32466,9 & 0,8 & 23598,0 & 0,6 \\
\hline México & 6015,0 & 2,5 & 23873,0 & 2,5 & 90647,5 & 2,3 & 87520,0 & 2,1 \\
\hline $\begin{array}{l}\text { Venezuela (Rep. } \\
\text { Bolivariana de) }\end{array}$ & $-1047,9$ & $-0,4$ & 1562,7 & 0,2 & 21426,0 & 0,5 & 13343,0 & 0,3 \\
\hline Asia y el Pacifico & 17737,5 & 7,4 & 117559,1 & 12,5 & 711497,1 & 17,7 & 595794,0 & 14,1 \\
\hline China & 0,1 & 0,0 & 16186,5 & 1,7 & 290427,0 & 7,2 & 254470,0 & 6,0 \\
\hline Corea, Rep. de & 1094,0 & 0,5 & 3305,0 & 0,4 & 24150,0 & 0,6 & 2673,0 & 0,6 \\
\hline Hong Kong, China & 2676,4 & 1,1 & 21332,7 & 2,3 & 90327,9 & 2,3 & 143041,0 & 3,4 \\
\hline India & 372,6 & 0,2 & 1047,5 & 0,1 & 15165,7 & 0,4 & 18775,0 & 0,4 \\
\hline Malasia & 3261,9 & 1,4 & 9647,4 & 1,0 & 48158,0 & 1,2 & 14642,0 & 0,3 \\
\hline Singapur & 3013,0 & 1,2 & 19068,0 & 2,0 & 83989,5 & 2,1 & 61818,0 & 1,5 \\
\hline Tailandia & 798,2 & 0,3 & 5098,6 & 0,5 & 31822,4 & 0,8 & 11199,0 & 0,3 \\
\hline Taiwán, prov. china & 658,7 & 0,3 & 4717,0 & 0,5 & 14591,0 & 0,4 & 12833,0 & 0,3 \\
\hline África & 10659,8 & 4,4 & 21621,5 & 2,3 & 61870,7 & 1,5 & 78742,0 & 1,9 \\
\hline \multicolumn{9}{|l|}{$\begin{array}{l}\text { Sudeste de Europa y } \\
\text { Comunidad de Estados }\end{array}$} \\
\hline Independientes (CEI) & $\ldots$ & $\ldots$ & 78,00 & 0,0 & 52212,0 & 1,3 & 92666,0 & 2,2 \\
\hline
\end{tabular}

Fuente: UNCTAD 2007. 
Gráfica 1. América Latina y el Caribe: entradas de IED y formación bruta de capital fijo, 1996-2006

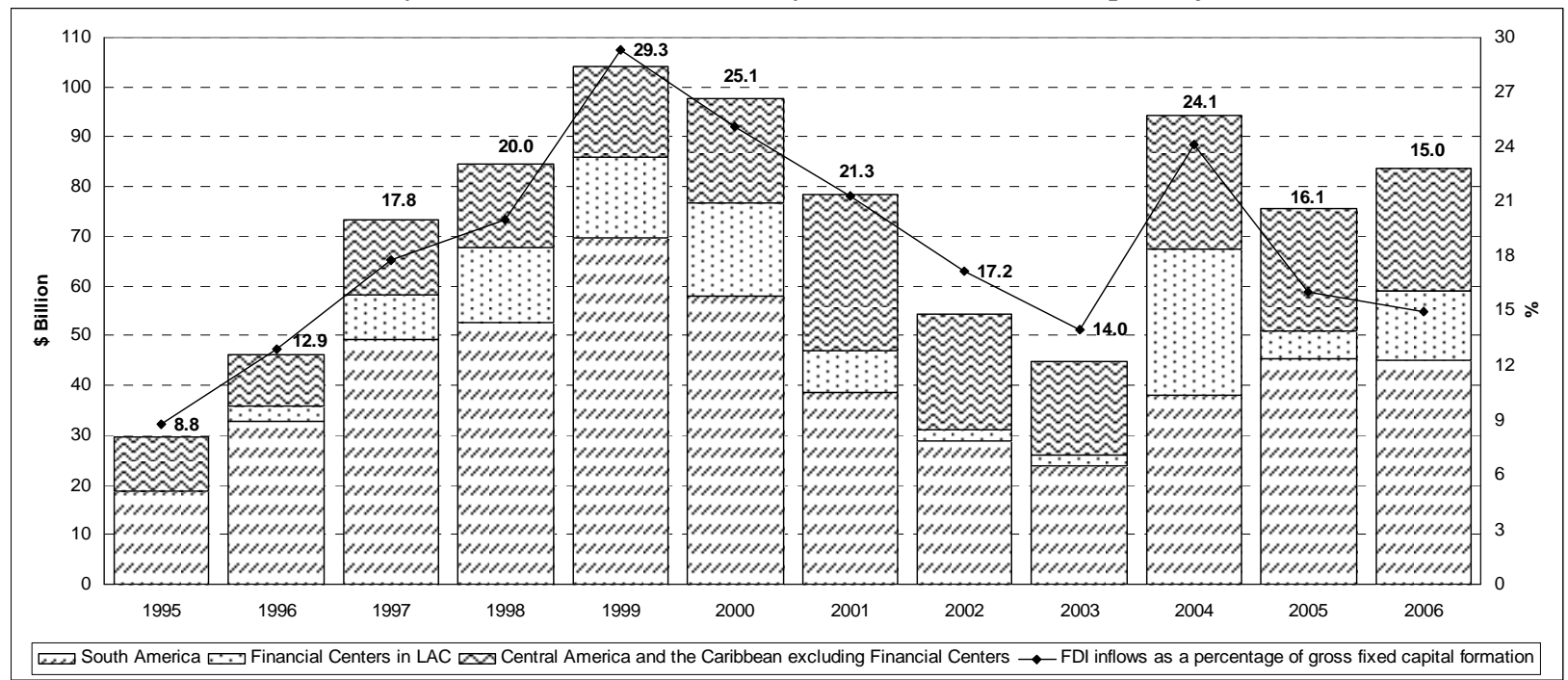

Fuente: UNCTAD, World Investment Report 2007.

Gráfica 2. América Latina y el Caribe: las diez economías más importantes en entradas de IED, 2005-2006 ${ }^{\mathrm{a}}$ (en miles de millones de dólares)

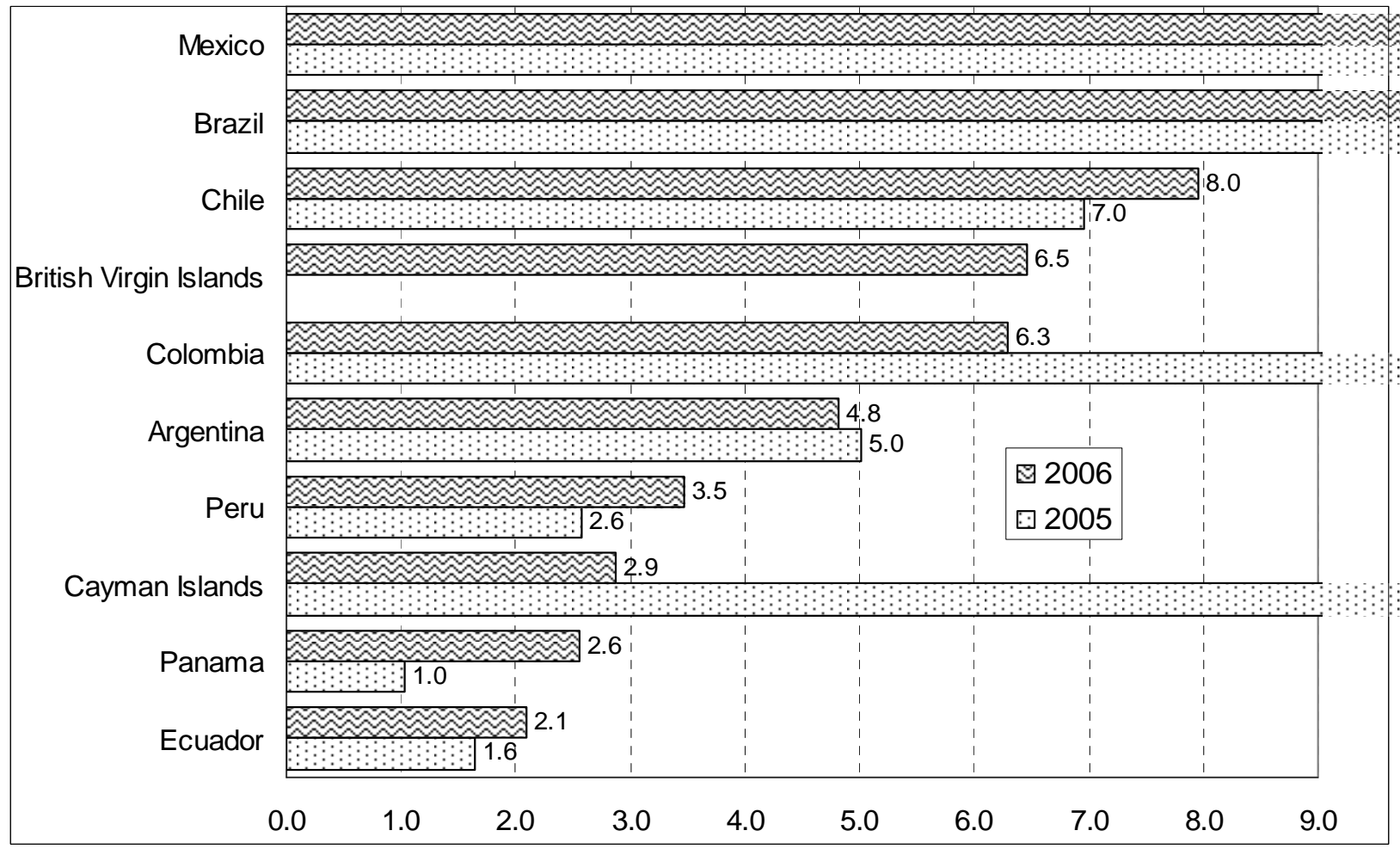

${ }^{a}$ Clasificadas en función de la magnitud de las entradas de IED en 2006.

Fuente: UNCTAD, World Investment Report 2007. 
Gráfica 3.

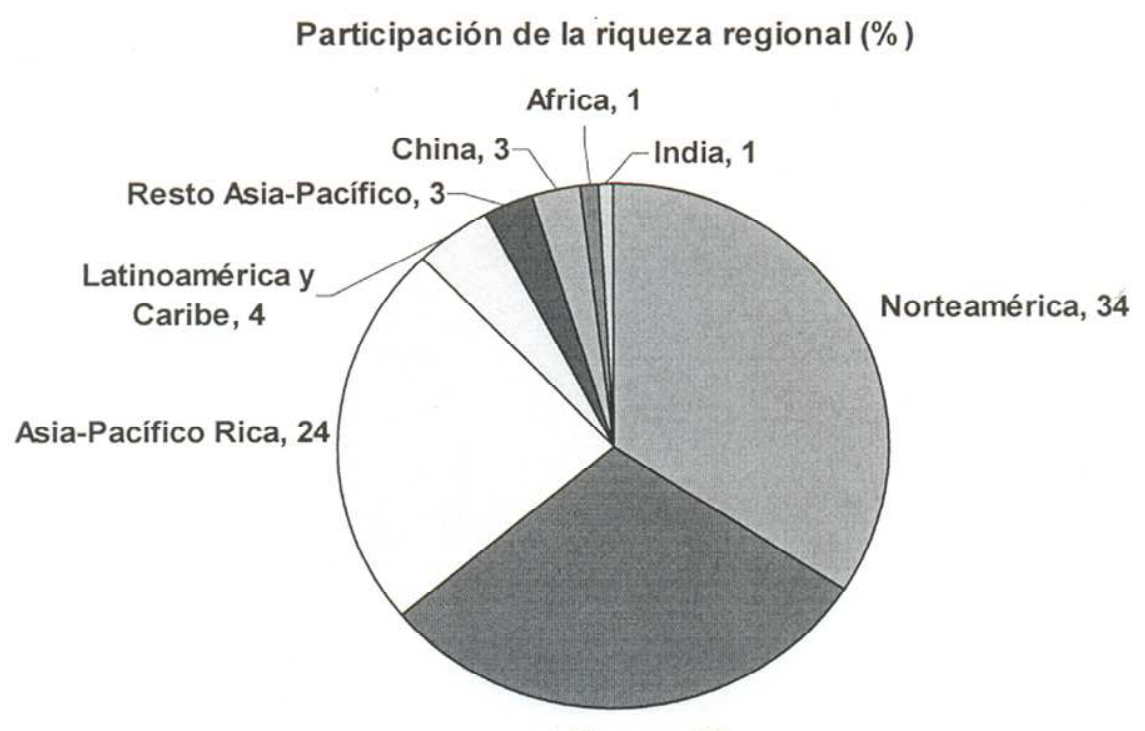

Europa, 30

Fuente: WIDER-ONU 2006.

Gráfica 4.

\section{Porcentaje membresía del $10 \%$ más rico}

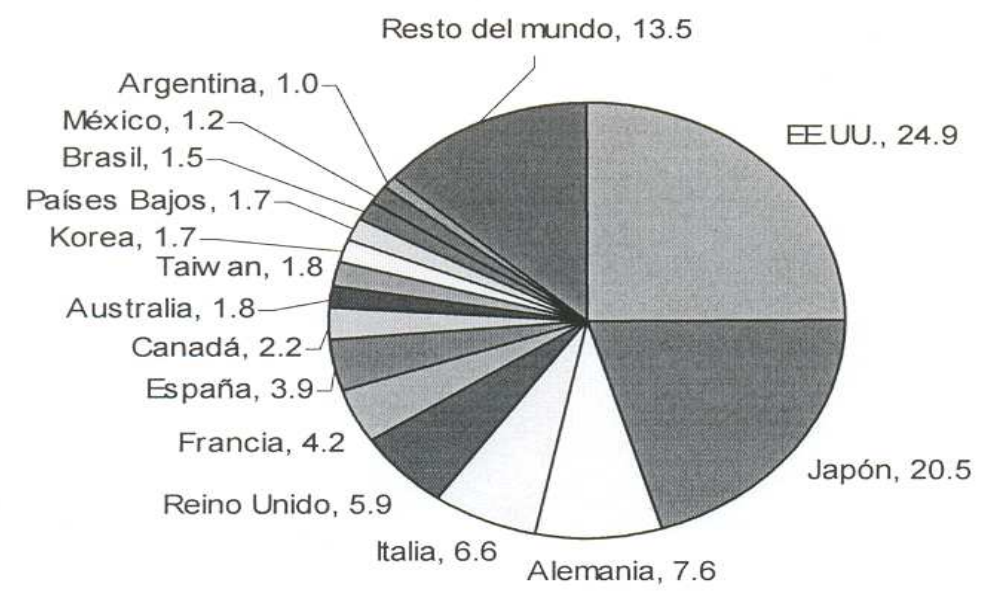

Fuente: WIDER-ONU 2006. 


\section{BIBLIOGRAFÍA}

Aguilar, Alonso, 2005, Economía Política del Desarrollo. IIE, Casa JuanPablos, México.

Amin, Samir, 2001, Crítica de nuestro tiempo. A los ciento cincuenta años del manifiesto comunista. S. XXI, México.

Boff, L. 2005, «La Contradicción capitalismo/ecología». En http://www.rebelion.org/noticia.php?id=35527

CEPAL, 2004, «Pobreza y distribución del ingreso». Panorama social de América Latina.

En http://www.eclac.cl/publicaciones/xml/6/20386/PSE2004_Cap1_Pobreza.pdf

Echeverría, Bolívar, 2005, Seminario: «La Modernidad: versiones y dimensiones». Mimeografiado. FF y L, UNAM, México.

2006, Vuelta de siglo. México, Era.

Ffrench, Ricardo, 2007, «América Latina después del Consenso de Washington». Quórum, núm. 18, otoño. Universidad de Alcalá, España.

Gandarilla, José, 2004, «¿De qué hablamos cuando hablamos de globalización». En Tercera vía y neoliberalismo, John Saxe-Fernández. CIICH-UNAM, México.

2005, América Latina en la conformación de la economía-mundo capitalista. Colección: El mundo Actual. CIICH-UNAM, México.

Girón, Alicia, 2005, Crisis financieras. Edición electrónica texto completo: http://www.eumed.net/libros/2005/agg/ 
Gunder, Frank, Andrés, 2005, Capitalismo y subdesarrollo en América Latina. En http://www.eumed.net/cursecon/textos/Frank/index.htm

Hinkelammert, Franz, 1996, El mapa del emperador. DEI, San José, Costa Rica.

Ianni, Octavio, 1999, Teorías de la globalización. CIICH-UNAM, S. XXI, México.

Ibarra, David, 2005, «La reconfiguración económica internacional». Economía UNAM, núm. 6, pp. 7-23, septiembre-diciembre. México.

Jalife-Rahme, Alfredo, 2005, «Bajo la lupa». En http://www.jornada.unam.mx/2005/03/30/020o1pol.php.

Lander, Edgardo, 2003, La colonialidad del saber: eurocentrismo y ciencias sociales. Perspectivas latinoamericanas. CLACSO, Buenos Aires, Argentina.

Maloney William F. y Perry Guillermo, 2005, «Hacia una política de innovación eficiente en América Latina». CEPAL, núm. 87, pp. 25-44, diciembre.

Martínez, Mariana, 2006, «Estados Unidos: Pobreza y desigualdad». En http://news.bbc.co.uk/hi/spanish/business/barometro_economico /newsid_5312000/5312126.stm

ONU, Centro de información para México, Cuba y República Dominicana. En http://www.cinu.org.mx/temas/desarrollo/dessocial/alimentos/ hambre.htm [consulta: mayo de 2008].

Oppenheimer, Andrés, 2005, «El desafío tecnológico para América Latina». En http://www.seguridad-la.com/artic/reflex/ref_8043.htm

Petras, James y Veltmeyer, Henry, 2003, La globalización desenmascarada. El imperialismo en el siglo XXI. Porrúa, México. 
Saxe-Fernández, John y N. R. Omar, 2001, «Globalización e imperialismo: la transferencia de excedentes de América Latina». En Globalización, Imperialismo y Clase Social, Saxe-Fernández et al. Lumen, Buenos Aires, Argentina.

UNCTAD, World Investment Report, 2007, «Informe sobre las inversiones en el mundo». En http://www.unctad.org/templates/webflyer.asp?docid=90018intltem id $=43618$ lang=1\&mode=dowloads.

WIDER- UNU, 2006, La distribución mundial de la riqueza de los hogares. World Institute for Development Economics of the Nations University. En http://www.wider.unu.edu/research/2006-2007/2006-2007-1/wider-wdhw

Xavier Sala-i-Martin, 1994, Apuntes de crecimiento económico. Antoni Bosch, Barcelona. 


\section{Notas}

${ }^{1}$ En esencia existen tres escenarios en el tema de la globalización: los defensores y beneficiarios, los adversarios, y los que se benefician pero al mismo tiempo son explotados.

${ }^{2}$ Revisar el informe de UNDP 2005.

${ }^{3}$ La decisión de utilizar en la elaboración de este artículo el concepto de globalización de la modernidad del mercado como predefinido, no ignora lo importante que es abundar la polémica sobre el tema del universalismo del mercado, sobre todo, revisando la posición de Hinkelammert 1996.

4 «... la dinámica de la globalización está en gran parte impulsada por el crecimiento masivo de los mercados financieros ... porque es este sector el que muestra el mayor incremento en volumen de flujos de capital y el que ha tenido menor efecto para estimular el crecimiento mundial de las fuerzas productivas» (Petras y Veltmeyer 2001: 61).

Considerada así a partir de los años ochenta, cuando para algunos historiadores inició la más reciente etapa del proceso globalizador del capitalismo.

${ }^{6}$ Xavier Sala-I-Martin, profesor de economía en Harvard, Yale, y Pompeu Fabra, ha hecho importantes aportes a la «teoría del crecimiento». Sus libros más conocidos son, entre otros: Apuntes de Crecimiento Económico (1994), Economic Growth, 2nd Edition (2003), Economía liberal para no economistas y no liberales (2002).

7 ... en la América Latina de hoy, entre $15 \%$ y $20 \%$ de la población comparte un estilo de vida de «Primer Mundo»:envían a sus hijos a escuelas privadas, pertenecen a los clubes de moda ... se hacen realizar tratamientos faciales ... viajan en automóviles de lujo por autopistas de cuota, al tiempo que se comunican por medio de computadoras, faxes y servicios privados de mensajería. Viven en fraccionamientos cercados protegidos por servicios de seguridad privados ... sus hijos asisten a universidades extranjeras ... forman parte de la red del nuevo sistema imperial. Y son ellos la audiencia a la que los presidentes dirigen sus grandilocuentes discursos sobre la entrada al Primer Mundo y la nueva era de prosperidad basada en el acomodo a los requerimientos del nuevo orden económico mundial (Saxe-Fernández y N. R. Omar 2001:237).

${ }^{8}$ Con esta figura quiero decir que a la economía global le es cada vez más difícil sostener su paso manteniendo las desigualdades e injusticias que le dan sustento económico y político a su proceso global.

9 «A la larga, es posible que la disposición de América Latina a depender pasivamente de la trasferencia de tecnología del extranjero y a no invertir lo suficiente en destrezas e investigación y desarrollo, I+D, haya sido un aspecto de la dependencia muchísimo más perjudicial que otros que han sido más objeto de estudios» (Maloney y Perry 2005:34).

${ }^{10}$ Una mirada más profunda de lo que significan las extracciones nos la ofrece Gandarilla al decir: «El proceso o la relación de explotación de unas regiones, países o clases por otras - evidenciada por el comportamiento de una serie de fuentes de trasferencia de excedentes internas y externas-, no solo mantiene una innegable actualidad, sino que ha experimentado un gran incremento y juega un papel importante en la explicación del drama contemporáneo a que han sido sometidos los países pobres y las clases pobres de estos países, a través de la aplicación de los programas de ajuste estructural y las políticas neoliberales»(Gandarilla 2005: 84).

${ }^{11}$ Fueron Brasil y México los que más dinero recibieron en la región con 19,000 millones de dólares cada uno. Importante es mencionar que el sector manufacturero es el que atrajo las mayores entradas, sobre todo en lo que respecta a la explotación de recursos naturales. Otro sector atractivo para los inversores extranjeros es el de productos básicos, por los altos precios registrados en el ámbito mundial. Es interesante destacar que los datos de IED para 2007 han llegado a la extraordinaria cifra de 105,925 millones de dólares, superior en $46 \%$ a la registrada el año anterior, sin embargo no sabemos aún el destino que tuvieron, aunque, con base en la experiencia de años anteriores, se puede pensar en dos caminos: uno, que se enfocaron principalmente a inversiones financieras y de servicios; dos, que a mayor recepción de capital ha correspondido siempre una mayor salida de beneficios.

${ }^{12}$ Para este principio revisar el libro de Lander, E. 2003.

13 Las medidas exigidas pretenden olvidar que, si bien es cierto la teoría del desarrollo continúa investigando sobre los mejores mecanismos para impulsar el desarrollo, hay una conclusión de la que puede estar orgullosa, y esta es que en la teoría y la práctica del desarrollo no hay reglas de oro. Por lo que promulga que las economías deben evitar la aplicación de medidas iguales para solucionar problemáticas aparentemente iguales.

${ }^{14}$ Entre 1999 y 2002, el proceso de superación de la pobreza no mostró avances significativos. De hecho ... aumentó 0,2 puntos porcentuales, mientras la indigencia registraba una alza de 0,9 puntos ... de manera que en términos absolutos en 2002 había —en América Latina- 21 millones más de personas pobres que 
en 1990, de los cuales 4 millones eran indigentes (CEPAL, 2004, «Pobreza y distribución del ingreso, Panorama social de América Latina», pp.51-95. En http://www.eclac.cl/publicaciones/xml/6/20386/PSE2004_Cap1_Pobreza.pdf.

15 ... una buena parte de las corrientes de IED hacia América Latina en las últimas décadas se debió al proceso de privatización que ha modernizado algunos sectores. Sin embargo, la privatización no es más que un cambio en la propiedad y no significa necesariamente un aumento de la capacidad productiva. (Ruth Ríos-Morales y David O’Donovan, 2006, «¿Pueden los países de América Latina y el Caribe emular el modelo irlandés para atraer inversión extranjera directa?». CEPAL, núm. 8, pp. 51-70, abril).

Fecha de recepción: 09 de mayo de 2008

Fecha de aceptación: 26 de mayo de 2008 